\title{
Optimized ventricular restraint therapy: Adjustable restraint is superior to standard restraint in an ovine model of ischemic cardiomyopathy
}

\author{
Lawrence S. Lee, MD, ${ }^{\text {a }}$ Ravi K. Ghanta, MD, ${ }^{a}$ Suyog A. Mokashi, MD, ${ }^{\text {a Otavio Coelho-Filho, MD, }}$ \\ Raymond Y. Kwong, MD, ${ }^{\mathrm{b}}$ Michael Kwon, MD, ${ }^{\mathrm{a}}$ Jian Guan, MS, ${ }^{\mathrm{c}}$ Ronglih Liao, $\mathrm{PhD},{ }^{\mathrm{c}}$ and \\ Frederick Y. Chen, MD, $\mathrm{PhD}^{\mathrm{a}}$
}

\begin{abstract}
Objective: The effects of ventricular restraint level on left ventricular reverse remodeling are not known. We hypothesized that restraint level affects the degree of reverse remodeling and that restraint applied in an adjustable manner is superior to standard, nonadjustable restraint.
\end{abstract}

\begin{abstract}
Methods: This study was performed in 2 parts using a model of chronic heart failure in the sheep. In part I, restraint was applied at control $(0 \mathrm{~mm} \mathrm{Hg}, \mathrm{n}=3)$, low $(1.5 \mathrm{~mm} \mathrm{Hg}, \mathrm{n}=3)$, and high $(3.0 \mathrm{~mm} \mathrm{Hg}, \mathrm{n}=3)$ levels with an adjustable and measurable ventricular restraint (AMVR) device. Restraint level was not altered throughout the 2-month treatment period. Serial restraint level measurements and transthoracic echocardiography were performed. In part II, restraint was applied with the AMVR device set at $3.0 \mathrm{~mm} \mathrm{Hg}(\mathrm{n}=6)$ and adjusted periodically to maintain that level. This was compared with restraint applied in a standard, nonadjustable manner using a mesh wrap $(n=6)$. All subjects were followed up for 2 months with serial magnetic resonance imaging.
\end{abstract}

Results: In part I, there was greater and earlier reverse remodeling in the high restraint group. In both groups, the rate of reverse remodeling peaked and then declined as the measured restraint level decreased with progression of reverse remodeling. In part II, adjustable restraint resulted in greater reverse remodeling than standard restraint. Left ventricular end diastolic volume decreased by $12.7 \%(P=.005)$ with adjustable restraint and by $5.7 \%(P=.032)$ with standard restraint. Left ventricular ejection fraction increased by $18.9 \%(P=.014)$ and $14.4 \%(P<.001)$ with adjustable and standard restraint, respectively.

Conclusions: Restraint level affects the rate and degree of reverse remodeling and is an important determinant of therapy efficacy. Adjustable restraint is more effective than nonadjustable restraint in promoting reverse remodeling. (J Thorac Cardiovasc Surg 2013;145:824-31)

Ventricular restraint therapy is a nontransplant surgical treatment for heart failure in which both ventricles are wrapped with prosthetic material. ${ }^{1,2}$ The intent is to mechanically constrain the ventricles without causing excessive diastolic constriction or tamponade. Numerous studies have demonstrated that restraint not only prevents further pathologic left ventricular (LV) dilatation but also

From the Divisions of Cardiac Surgery ${ }^{\mathrm{a}}$ and Cardiology ${ }^{\mathrm{b}}$ and the Cardiac Muscle Research Laboratory, ${ }^{\mathrm{c}}$ Division of Cardiology, Brigham and Women's Hospital, Harvard Medical School, Boston, Mass.

Funded by Brigham and Women's Hospital, Department of Surgery (to F.Y.C.), Brigham and Women's Hospital, Cardiac Surgery Research Fund (to R.M.B.), J. Paul Finnegan Endowment in Cardiac Surgery Research (to F.Y.C.), National Institutes of Health T32HL076130 (to L.S.L.), F32HL080904 (to R.K.G.), F32HL104923 (M.K.), RO1HL091157 (to R.Y.K.), RO1HL10378 (to R.L.), RO1HL102791 (to R.L.), RO1HL102786 (to R.L.), and RO1HL090862 (to F.Y.C.). The Brigham and Women's Hospital has patent rights on the device described in this manuscript.

Disclosures: Authors have nothing to disclose with regard to commercial support.

Received for publication Jan 19, 2012; revisions received April 9, 2012; accepted for publication May 9, 2012; available ahead of print June 14, 2012.

Address for reprints: Frederick Y. Chen, MD, PhD, Division of Cardiac Surgery, Brigham and Women's Hospital, 75 Francis St, Boston, MA 02115 (E-mail: fchen@partners.org).

0022-5223/ $\$ 36.00$

Copyright (c) 2013 by The American Association for Thoracic Surgery

doi: $10.1016 /$ j.jtcvs.2012.05.018 induces reverse remodeling at both the molecular and systemic levels. $^{3-6}$

Restraint devices tested in clinical trials, however, do not allow for either the measurement or adjustment of wrap tightness; the restraint level is not quantified. Such restraint devices are applied around the heart at a subjective level of tightness. The influence of restraint level on therapy efficacy is thus unclear. To address this issue, we developed and described a new technique-adjustable and measurable ventricular restraint (AMVR) - in which a fluid-filled epicardial balloon is placed around the heart. ${ }^{7}$ By use of a fluid layer to effect restraint, adjustability and measurability of therapy was achieved.

We demonstrated with AMVR that restraint decreases LV transmural myocardial pressure and indices of myocardial oxygen consumption in a restraint level-dependent manner. ${ }^{7}$ Progressively higher levels of restraint will ultimately cause tamponade, however, and we showed that this tamponade physiology is secondary to (RV) constriction only. ${ }^{8}$ When restraint is applied in a fixed, constant manner, our results demonstrate that the rate of reverse remodeling slows as the heart shrinks and undergoes reverse remodeling. These findings suggest that the interplay between restraint 


\section{Abbreviations and Acronyms \\ AMVR $=$ adjustable and measurable ventricular restraint \\ $\mathrm{BNP}=$ brain natriuretic peptide \\ $\mathrm{CMR}=$ cardiac magnetic resonance \\ $\mathrm{EDV}=$ end-diastolic volume \\ $\mathrm{EF} \quad=$ ejection fraction \\ ESV $=$ end-systolic volume \\ $\mathrm{LV}=$ left ventricular \\ MMP-2 = myocardial matrix metalloproteinase-2 \\ $\mathrm{RV} \quad=$ right ventricular}

therapy and ventricular remodeling is a dynamic process that may require periodic adjustment of restraint level to maintain therapeutic efficacy.

In this study, we seek to definitively investigate the role that restraint level plays in inducing reverse remodeling. Although our previous studies delineated the relationship between restraint level and acute ventricular mechanical indices, these were not long-term in vivo studies evaluating the relationship between restraint level and reverse remodeling itself. For the present study, we hypothesized that (1) the initial, or starting, restraint level is a key determinant of the degree of reverse remodeling in standard, nonadjusted restraint therapy and (2) restraint therapy applied in an optimized, adjustable manner is superior to restraint applied in a fixed, nonadjustable manner in promoting reverse remodeling.

\section{METHODS}

\section{Study Design Overview}

This study was performed in 2 parts: (1) a 4-month study investigating the effects of different initial restraint levels on reverse remodeling and (2) a 4-month study comparing optimized, adjustable restraint therapy to standard, nonadjustable restraint therapy.

In part I, 9 sheep underwent coronary artery ligation through a left thoracotomy to develop heart failure. Animals were then divided into 3 equal groups: control (no restraint), low restraint $(1.5 \mathrm{~mm} \mathrm{Hg})$, and high restraint (3.0 mm Hg). Nine total sheep were used. The control group had no restraint device implanted. Low and high restraint groups underwent reoperative median sternotomy with implantation of the AMVR device. The restraint device was filled with fluid to achieve the desired restraint levels ( $1.5 \mathrm{~mm} \mathrm{Hg}$ in the low group and $3.0 \mathrm{~mm} \mathrm{Hg}$ in the high group). Restraint level was defined by the measured balloon luminal pressure at end-diastole. The levels chosen were based on our previous results with this animal model demonstrating beneficial changes at these restraint levels. Restraint levels greater than $3.0 \mathrm{~mm} \mathrm{Hg}$ were not tested because our previous results demonstrated decreased cardiac output with impaired RV filling at those higher levels, suggesting that $3.0 \mathrm{~mm} \mathrm{Hg}$ is the optimal restraint level for this particular model. ${ }^{7,8}$

After device placement, all subjects were followed up for 2 months with serial transthoracic echocardiography. The volume of fluid in the AMVR device was not altered to reflect the unchanging nature of clinical devices. Restraint level was measured weekly throughout the study period to assess the effect of reverse remodeling on restraint level. A terminal experiment was performed to obtain and analyze myocardial tissue samples for molecular markers of remodeling. The long-term effects of the initial starting restraint level on ventricular volumes and the level of restraint itself were then measured and compared.

In part II, 12 sheep underwent coronary artery ligation, and heart failure developed by 2 months postoperatively in all of them. Animals were then divided into 2 groups: standard, nonadjustable restraint $(n=6)$ and optimized, adjustable restraint $(n=6)$. All sheep underwent reoperative median sternotomy for implantation of the assigned treatment device. In the standard restraint group, a polypropylene mesh was wrapped around the heart to simulate unchanging restraint devices. In the adjustable restraint group, AMVR devices were implanted and set at a restraint level of 3.0 $\mathrm{mm} \mathrm{Hg}$. This level was chosen because our previously published studies showed this to be the optimal restraint level at which beneficial changes in ventricular mechanical indices are maximized while adverse systemic hemodynamic effects are minimized in this particular sheep model. ${ }^{7,8}$

All 12 subjects were then followed up for 2 months with serial cardiac magnetic resonance (CMR) imaging. In the adjustable restraint group, the restraint level was measured biweekly and the volume in the restraint device adjusted to maintain a restraint pressure of $3.0 \mathrm{~mm} \mathrm{Hg}$ throughout the study duration. No postoperative measurement or manipulation of restraint level was possible in the fixed restraint group simulating standard clinical restraint. Blood samples were obtained biweekly in all animals to assess for plasma markers of remodeling. A terminal experiment was performed to obtain and analyze myocardial tissue samples for molecular markers of remodeling.

A total of 21 (9 in part I, 12 in part II) adult male sheep (30-40 kg) were used for the 2 parts of this study. All animals received humane care in compliance with the "Guide for Care and Use of Laboratory Animals" published by the National Institutes of Health (NIH publication no. 86-23, revised 1996). The protocol was approved by the Institutional Animal Care and Use Committee of Harvard Medical School.

The specific techniques and methodologies used are described below.

Heart failure model. An ovine model of postinfarction heart failure involving ligation of the first and second diagonal coronary arteries was used. $^{7-9}$ Heart failure, defined as an LV ejection fraction (EF) less than $40 \%$ and a $100 \%$ increase in LV end-diastolic volume (EDV) from preoperative baseline, was confirmed by either echocardiography or CMR imaging.

Adjustable and measurable ventricular restraint. The AMVR device is a half-ellipsoidal balloon constructed from medicalgrade polyurethane sheets (Polyzen, Inc, Apex, NC) and has been described previously. ${ }^{7}$ Each balloon is composed of 2 layers. An access line placed between the 2 layers allows for pressure measurement inside the balloon lumen and the addition or withdrawal of fluid. Because the outer layer of the balloon is relatively nonstretchable, fluid introduced into the balloon lumen has only 1 direction of filling space-inward toward the heart, thereby creating a tighter wrap. Conversely, withdrawal of fluid from the balloon lumen creates a looser wrap. The device is placed around the heart via median sternotomy and sutured to the atrioventricular groove such that both ventricles are covered. The balloon access line is connected to an implantable catheter (Port-a-Cath; Bard Medical, Covington, Ga), which is placed in the chest wall. The port can be accessed with an 18gauge Huber needle and connected to a Statham P10EZ pressure transducer (SpectraMed, Oxford, Calif). Measuring the pressure within the balloon when the heart is largest in size-end-diastole-allows wrap tightness (or restraint level) to be precisely quantified. Restraint level is changed by adding or removing fluid from the balloon while luminal pressure is monitored in real time.

Standard, nonadjustable ventricular restraint. Standard, fixed restraint intended to simulate clinically tested restraint devices was achieved with a polypropylene mesh wrap (Ethicon, Inc, Somerville, $\mathrm{NJ})$. A median sternotomy was performed and the mesh was placed around the heart in accordance with guidelines used for placement of the Acorn CorCap cardiac support device (Acorn Cardiovascular, Inc, St Paul, 
Minn): the mesh was wrapped around the heart and secured to the atrioventricular groove such that LV EDV decreased by $5 \%$ on intraoperative epicardial echocardiography. ${ }^{10,11}$ No measurement or adjustment of restraint level was possible in the postoperative period.

Transthoracic echocardiography. In part I, quantitative 2-dimensional transthoracic echocardiography (Cypress Acuson; Siemens Medical Solutions, Malvern, $\mathrm{Pa}$ ) with a $3.5-\mathrm{MHz}$ probe was performed before infarction and then weekly in all animals. Parasternal long-axis images were obtained by placing the ultrasound probe along the fourth through seventh intercostal spaces with the animal in the upright, standing position. Stroke volume was calculated as the difference in LV EDV and LV endsystolic volume (ESV), and LV EF was calculated as the ratio of stroke volume to LV EDV.

CMR imaging. In part II, CMR imaging was performed in all subjects 8 weeks after coronary ligation (but before restraint device implantation) to confirm development of heart failure and then monthly after restraint device placement (Figure 1). Imaging was performed with a 3-Tesla magnetic resonance scanner (Signa CV/i; General Electric Healthcare, Milwaukee, Wis). All acquisitions were obtained with an 8 -element cardiac phasedarray surface coil with the animal in the lateral decubitus position under general anesthesia and proper ventilation. Peripheral pulse gating and breath holding were used as much as possible to minimize cardiac and respiratory motion, respectively. Details of the CMR sequences and imaging parameters have been published previously. ${ }^{8}$

Serum brain natriuretic peptide measurement. Relative concentrations of serum brain natriuretic peptide (BNP) were determined using a modified Dotblot technique. A $2-\mu \mathrm{L}$ portion of each serum sample was placed onto a $0.2-\mu \mathrm{m}$ nitrocellulose membrane (catalog no. 162-0168;

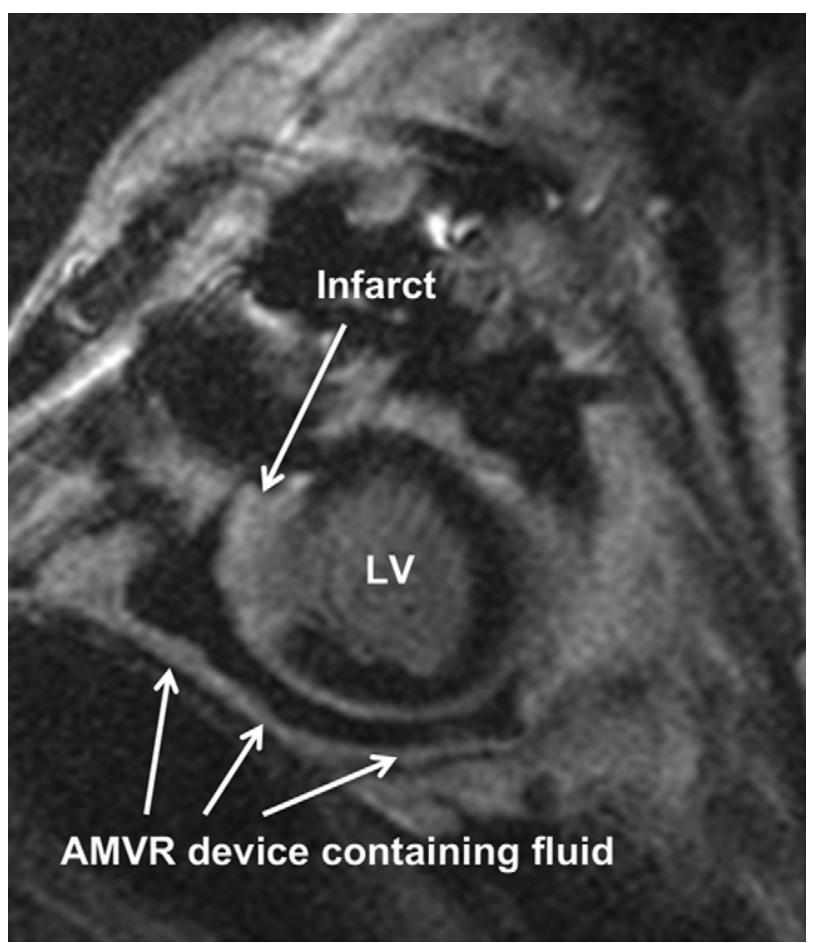

FIGURE 1. Representative cardiac magnetic resonance imaging shortaxis section (delayed enhancement series) of heart failure ovine subject. Infarcted area is white and identified by upper arrow. The adjustable and measurable ventricular restraint $(A M V R)$ device is identified by lower arrows and the fluid within the device can be clearly seen as black. The AMVR device surrounds the entire heart but is only partially seen here owing to the cut of this particular image. $L V$, Left ventricle.
Bio-Rad Laboratories, Hercules, Calif). After drying, the membranes were primed in $50 \mathrm{~mL}$ of a $5 \%$ bovine serum albumin (Sigma-Aldrich, St Louis, $\mathrm{Mo}$ ) in $1 \mathrm{X}$ phosphate-buffered saline (GIBCO/Invitrogen, Carlsbad, Calif) followed by incubation in a 1:1000 solution of primary rabbit anti-BNP polyclonal antibody (catalog no. BML BA1117; Bio-Mol Intl., Plymouth Meeting, Pa). The membranes were washed in $1 \mathrm{X}$ phosphate-buffered saline with $1 \%$ Tween followed by dark room incubation with a 1:3000 fluorescent secondary antibody solution (Alexa-Fluor 680 GoRRRat Anti-Rabbit immunoglobulin G, catalog no. A21109; Invitrogen). Each sample was analyzed for the intensity of the 700-nm infrared signal over circular areas surrounding the dot at 3.5 intensity (Odyssey Imager; LiCor Biosciences, Lincoln, Neb).

Myocardial matrix metalloproteinase-2 measurement. At the end of the experimental period, all animals underwent terminal study and explantation of the heart. The infarcted area was identified and the LV bisected. Tissue samples were taken from 3 zones: infarct (identified by whitish fibrotic discoloration), border (defined as tissue located between the outer border of the infarct and $5 \mathrm{~mm}$ outward beyond that border), and normal (remote, pink, and healthy appearing LV). All samples were flash frozen in liquid nitrogen and stored at $-80^{\circ} \mathrm{C}$. Myocardial matrix metalloproteinase-2 (MMP-2) levels were analyzed using a Western blot technique as described below.

Protein extraction and determination of total protein concentration. Tissue samples were ground with a mortar and pestle, suspended in $200 \mu \mathrm{L}$ of lysis buffer ( $20 \mathrm{mmol} / \mathrm{L}$ TRIS-HCl-based Cell Lysis Buffer, catalog no. 9803; Cell Signaling Technology, Danvers, Mass) containing $1 \mathrm{X}$ phenylmethanesulfonylfluoride, and sonicated at $35 \%$ efficiency. After centrifugation at $13,000 \mathrm{rpm}$ for 20 minutes at $4{ }^{\circ} \mathrm{C}$, the supernatant was diluted 10:1. The protein concentration for each sample was determined by incubating $25 \mu \mathrm{L}$ of the diluted supernatant with $125 \mu \mathrm{L}$ of alkaline copper tartrate solution (Reagent A, catalog no. 500-0113; Bio-Rad) and $1000 \mu \mathrm{L}$ of dilute Folin reagent (Reagent B, catalog no. 500-0114; Bio-Rad). The absorbance of each sample at $595 \mathrm{~nm}$ was measured by spectrophotometry. The protein concentration (in micrograms per milliliter) was determined by comparing these absorbance values to that of a protein standard.

Protein electrophoresis and transfer. The calculated volume of each sample containing $50 \mu \mathrm{g}$ of total protein was added to $12 \mu \mathrm{L}$ of $2 \mathrm{X}$ loading buffer (Laemmly Sample Buffer, catalog no. 161-0737; Bio$\mathrm{Rad}$ ), $2 \mu \mathrm{L}$ of beta-mercaptoethanol (catalog no. 161-0710; Bio-Rad), and cell lysis buffer and then incubated at $95^{\circ} \mathrm{C}$ for 10 minutes. Electrophoresis was performed using $20 \mu \mathrm{L}$ of each sample in precast gels (4\%-12\% Criterion XT Bis-Tris precast polyacrylamide gel, catalog no. 345-0124; Bio-Rad) with running buffer (Criterion XT MOPS Running Buffer, cata$\log$ no. 161-0788; Bio-Rad). Protein transfer onto a $0.45-\mu \mathrm{m}$ pore size polyvinylidene difluoride transfer membrane (Immobilon-P PVDF Transfer Membrane, catalog no. IPVH08310; Millipore, Billerica, Mass) in TRIS/ glycine buffer (catalog no. 161-0771; Bio-Rad) was performed. Membranes were then incubated with a 1:1000 rabbit anti-MMP-2 antibody solution (gift from Dr William Stetler-Stevenson of the National Institutes of Health/National Cancer Institute), followed by dark-room incubation with a 1:3000 fluorescent secondary antibody solution (Alexa-Fluor 680 Goat Anti-Rabbit immunoglobulin G, catalog no. A21109; Invitrogen). Each band was analyzed at 3.5 intensity for the intensity of the 700-nm infrared signal over the surrounding rectangular area.

\section{Statistics}

All data are expressed as mean \pm standard error. Mean values of parameters between groups were compared using a general linear mixed model. Of the 144 data points collected, $8(5.6 \%)$ were missing at random owing to sporadic equipment malfunction or unavailability (such as the CMR magnet, imaging analysis software, or infrared scanner). These missing values were imputed using the linear trend at point method. Repeated measures 
TABLE 1. LV volumes for all subjects in part I

\begin{tabular}{|c|c|c|c|c|c|c|c|}
\hline & \multicolumn{3}{|c|}{ LV EDV (mL) } & \multicolumn{2}{|c|}{ LV ESV (mL) } & \multicolumn{2}{|c|}{ LV EF (\%) } \\
\hline & Pre & Post & Mean $\Delta$ & Pre & Post & Pre & Post \\
\hline Control & $110( \pm 13)$ & $165^{*}( \pm 6)$ & $+52 \%( \pm 16)$ & $82( \pm 16)$ & $115^{*}( \pm 8)$ & $26( \pm 6)$ & $23( \pm 8)$ \\
\hline Low restraint & $129( \pm 7)$ & $106^{*}( \pm 11)$ & $-18 \% \dagger( \pm 4)$ & $94( \pm 13)$ & $61 *( \pm 10)$ & $28( \pm 5)$ & $41 *( \pm 9)$ \\
\hline High restraint & $133( \pm 19)$ & $95^{*}( \pm 13)$ & $-28 \% \dagger( \pm 2)$ & $95( \pm 12)$ & $67 *( \pm 16)$ & $28( \pm 3)$ & $44 *( \pm 9)$ \\
\hline
\end{tabular}

Left ventricular $(L V)$ size and function changes with adjustable and measurable ventricular restraint. $E D V$, End-diastolic volume; $E S V$, end-systolic volume; $E F$, ejection fraction; Pre, time point of adjustable and measurable ventricular restraint device implant; Post, after 2 months of therapy. $* P<.05$ for change within subjects from Pre to Post therapy. $\dagger P<.05$ for difference in percentage change from Pre to Post therapy between low and high restraint subjects.

data were assessed using a repeated measures design to control for the interrelatedness of our outcomes. Statistics were performed using SPSS 13.0 (SPSS, Inc, Chicago, Ill).

\section{Anesthesia and Postoperative Care}

For all operative procedures, we used standard perioperative and postoperative methods previously described. ${ }^{7,8}$

\section{RESULTS}

\section{Part I: Long-Term Effects of Initial Restraint Level}

Ventricular size and function. Ventricular volume data are listed in Table 1. Figure 2 summarizes the effects of initial restraint level on LV size and function. In control subjects in which no restraint was applied, the LV continued to enlarge while EF declined. In both treatment groups, LV EDV and ESV decreased and EF improved. The degree of improvement in LV volumes correlated to the level of restraint therapy applied, with high restraint leading to greater decreases in LV volume than low restraint. After 8 weeks of treatment, LV EDV decreased by an average of $18.2 \%$ in the low restraint group $(P=.005)$ and by $28.4 \%$ in the high restraint group $(P=.012)$, whereas LV EF improved from $28 \%$ to $41 \%$ in the low restraint group $(P=.012)$ and from $28 \%$ to $44 \%$ in the high restraint group $(P=.03)$. Rate of reverse remodeling. Both restraint groups displayed reductions in LV EDV, with high restraint resulting

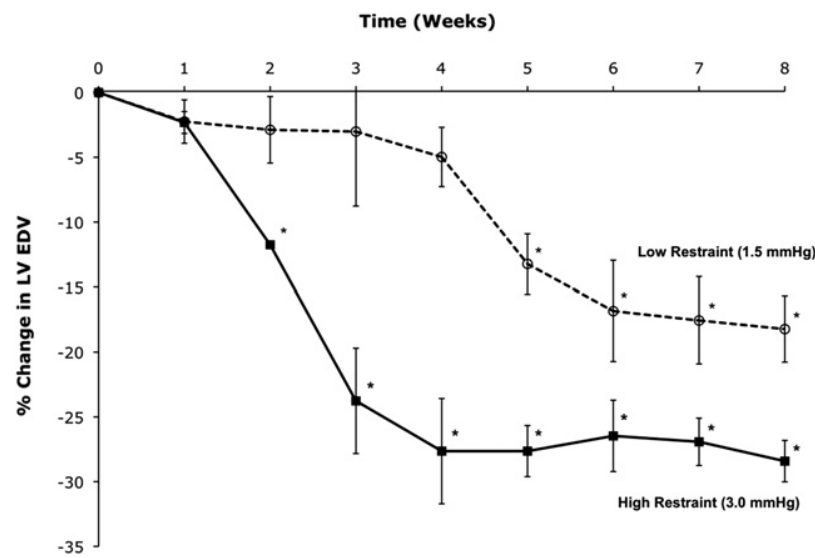

FIGURE 2. Mean change in left ventricular end-diastolic volume ( $L V$ $E D V)$ after restraint device implantation for the low and high restraint groups. $* P<.05$ for change in LV EDV from time zero (start of restraint therapy). in a statistically significant decrease in LV EDV occurring earlier than in low restraint (Figure 2). The rate of reverse remodeling (ie, the rate of change in LV EDV per week) was not constant for the 2 groups, as shown in Figure 3: the rate peaked at 21 days for high restraint and at 35 days for low restraint. After these time points, reverse remodeling subsequently slowed in both groups.

Effect of reverse remodeling on measured restraint level. As reverse remodeling occurred and LV EDV decreased, measured restraint level decreased in both restraint groups. The high restraint group maintained the initial restraint level (ie, $3.0 \mathrm{~mm} \mathrm{Hg}$ as set at the time of AMVR device implantation) longer than did the low restraint group: in the high restraint group, the measured restraint level did not begin to fall until LV EDV decreased by $13 \%$, whereas in the low restraint group, the measured restraint level began to decrease after a $4 \%$ reduction in LV EDV.

\section{Part II: Optimized, Adjustable Restraint Versus Standard, Nonadjustable Restraint}

Ventricular size and function. Volume and hemodynamic data results are listed in Table 2. LV volumes decreased and LV EF increased in both treatment groups (Figure 4, $A$ and $B$ ).

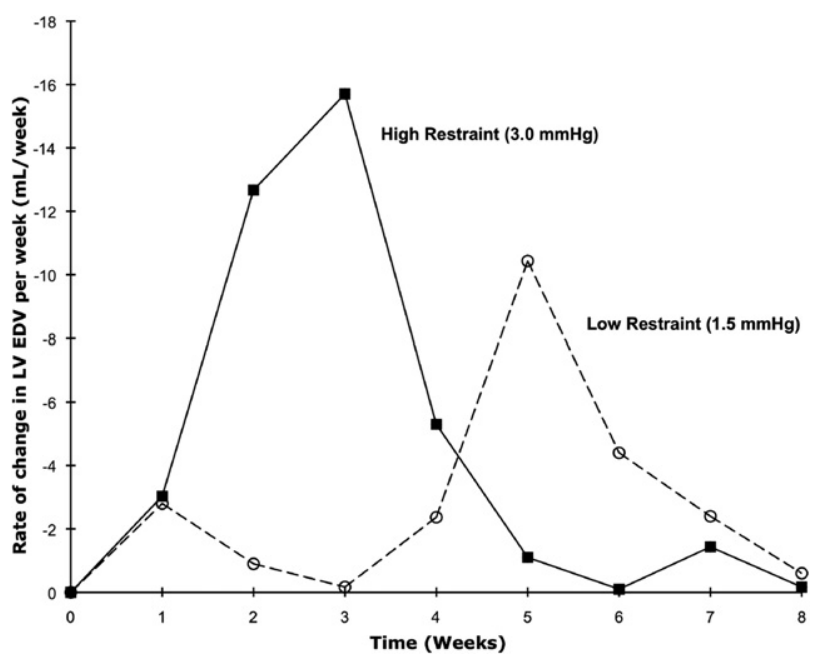

FIGURE 3. Rate of reverse remodeling (change in left ventricular enddiastolic volume [ $L V E D V]$ per week) for the low and high restraint groups in part I. 
TABLE 2. LV volume data for subjects in part II

\begin{tabular}{|c|c|c|}
\hline & $\begin{array}{l}\text { Standard restraint } \\
(\mathrm{n}=6)\end{array}$ & $\begin{array}{c}\text { Adjustable restrain } \\
(\mathrm{n}=6)\end{array}$ \\
\hline \multicolumn{3}{|c|}{ LV EDV (mL) } \\
\hline Pre & $106( \pm 11)$ & $150( \pm 31)$ \\
\hline Post & $99^{*}, \dagger( \pm 8)$ & $130^{*}, \dagger( \pm 25)$ \\
\hline \multicolumn{3}{|c|}{ LV ESV (mL) } \\
\hline Pre & $70( \pm 9)$ & $102( \pm 24)$ \\
\hline Post & $62^{*}, \dagger( \pm 8)$ & $81^{*}, \dagger( \pm 17)$ \\
\hline \multicolumn{3}{|c|}{$\begin{array}{l}\text { LV EDV indexed to body } \\
\text { weight }(\mathrm{mL} / \mathrm{kg})\end{array}$} \\
\hline Pre & $2.1( \pm 0.3)$ & $3.4( \pm 0.7)$ \\
\hline Post & $1.7^{*}, \dagger( \pm 0.3)$ & $2.4^{*}, \dagger( \pm 0.7)$ \\
\hline \multicolumn{3}{|c|}{$\begin{array}{l}\text { LV ESV indexed to body } \\
\text { weight }(\mathrm{mL} / \mathrm{kg})\end{array}$} \\
\hline Pre & $1.3( \pm 0.2)$ & $2.3( \pm 0.5)$ \\
\hline Post & $1.0^{*}, \dagger( \pm 0.2)$ & $1.5^{*}, \dagger( \pm 0.4)$ \\
\hline \multicolumn{3}{|c|}{ LV EF $(\%)$} \\
\hline Pre & $33.8( \pm 3.2)$ & $32.4( \pm 3.1)$ \\
\hline Post & $38.4^{*}( \pm 4.8)$ & $38.3^{*}( \pm 1.8)$ \\
\hline \multicolumn{3}{|c|}{ Stroke volume (mL) } \\
\hline Pre & $35( \pm 4)$ & $48( \pm 9)$ \\
\hline Post & $38( \pm 3)$ & $50( \pm 9)$ \\
\hline \multicolumn{3}{|c|}{$\begin{array}{l}\text { Stroke volume indexed to } \\
\text { body weight }(\mathrm{mL} / \mathrm{kg})\end{array}$} \\
\hline Pre & $0.7( \pm 0.1)$ & $1.0( \pm 0.2)$ \\
\hline Post & $0.7( \pm 0.1)$ & $0.9( \pm 0.3)$ \\
\hline \multicolumn{3}{|c|}{ Cardiac output (L/min) } \\
\hline Pre & $3.7( \pm 1.2)$ & $4.9( \pm 1.3)$ \\
\hline Post & $4.2( \pm 1.0)$ & $5.4( \pm 2.3)$ \\
\hline \multicolumn{3}{|c|}{ Body weight $(\mathrm{kg})$} \\
\hline Pre & $51( \pm 7)$ & $48( \pm 5)$ \\
\hline Post & $57( \pm 5)$ & $55( \pm 2)$ \\
\hline
\end{tabular}

Left ventricular $(L V)$ size and function changes with adjustable and standard restraint. $E D V$, End-diastolic volume; $E S V$, end-systolic volume; $E F$, ejection fraction; Pre, time point of restraint device implant; Post, after 2 months of therapy. ${ }^{*} P<.05$ for change within treatment group from Pre to Post therapy. $\nmid P<.05$ for difference in change from Pre to Post therapy between standard and adjustable restraint groups.

After 8 weeks of therapy, in the adjustable restraint group, LV EDV decreased by $12.7 \%(P=.005)$, LV ESV decreased by $19.0 \%(P<.001)$, and LV EF increased by $18.2 \%(P=.014)$. In the standard restraint group, LV EDV decreased by $5.7 \%(P=.032)$, LV ESV decreased by $9.4 \%(P=.001)$, and LV EF increased by $13.6 \%$ $(P<.001)$. $\mathrm{LV}$ ventricular volumes were indexed to animal body weight (LV EDV index and LV ESV index) to account for any potential effects of animal growth during the study period. These indexed volumes also decreased significantly $(P<.05)$ for both restraint groups. The difference in change between the adjustable and standard restraint groups was statistically significant for LV EDV $(P=.006), \mathrm{LV}$ ESV $(P=.001)$, LV EDV index $(P=.009)$, and LV ESV index $(P=.003)$. RV EDV and RV EF showed no significant change in either group throughout the study period: RV EDV increased by $5 \%(P=.1)$ and RV EF had no change $(P=.87)$ in the adjustable group, whereas RV EDV
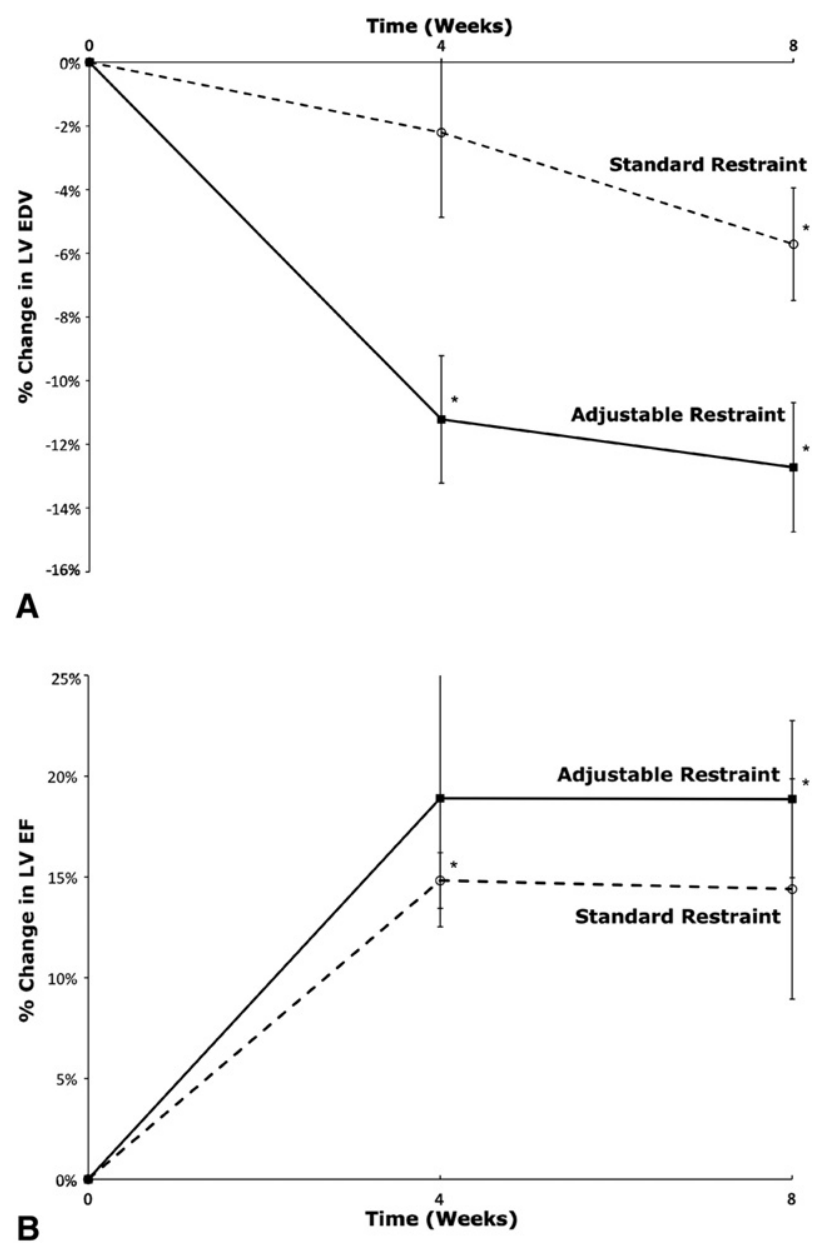

FIGURE 4. Mean change in left ventricular end-diastolic volume ( $L V$ $E D V)(\mathrm{A})$ and left ventricular ejection fraction $(L V E F)(\mathrm{B})$ after restraint device implantation for the adjustable and standard restraint groups in part II. $* P<.05$ for change in LV EDV from time zero (start of restraint therapy).

increased by $1.2 \%(P=.72)$ and RV EF increased by $1.6 \%(P=.79)$ in the standard group.

Plasma BNP. Coronary artery ligation resulted in an average rise in plasma BNP of $37 \%$ by the eighth postoperative week in all subjects. After restraint device placement, plasma BNP levels subsequently fell in both groups (Figure 5). By the end of the 2-month treatment period, the plasma BNP level decreased by $12.6 \%$ (when compared with the level at time of restraint device implantation) with adjustable restraint $(P=.47)$ and by $4.5 \%$ with standard restraint $(P=.64)$. There was no significant difference between the adjustable and standard groups $(P=.38)$.

Myocardial tissue MMP-2. In each subject, the infarct zone had significantly higher levels of MMP-2 than either the border or normal zones $(P=.02)$. Among the different groups, the control group displayed substantially higher levels of MMP-2 in all tissue zones (infarct, border, and normal) than both restraint groups (Figure 6). Between the restraint groups, 


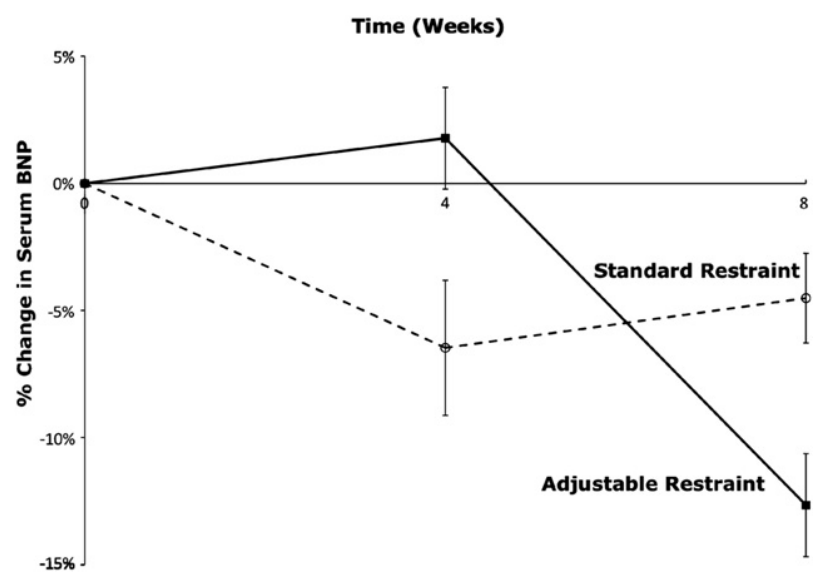

FIGURE 5. Plasma brain natriuretic peptide $(B N P)$ levels in the adjustable and standard restraint groups.

the adjustable group had slightly lower levels of MMP-2, particularly in the border and infarct zones, than the standard group, but this was not statistically significant $(P=.6)$.

\section{DISCUSSION}

Ventricular restraint therapy is a promising surgical option for heart failure. Studies using the Acorn CorCap Cardiac Support Device (Acorn Cardiovascular, Inc) and the Paracor Heartnet Device (Paracor Medical Systems, Sunnyvale, Calif) have shown restraint to be one of the few therapies that can reverse pathologic LV remodeling in both animal models and patients. ${ }^{1-5,12,13}$

Further optimization of this therapy, however, will require a comprehensive understanding of the precise relationship between the restraint level applied and the reverse remodeling engendered. By using our adjustable device in a sheep model, $\mathrm{we}^{7}$ previously demonstrated that restraint level has a direct effect on ventricular mechanics

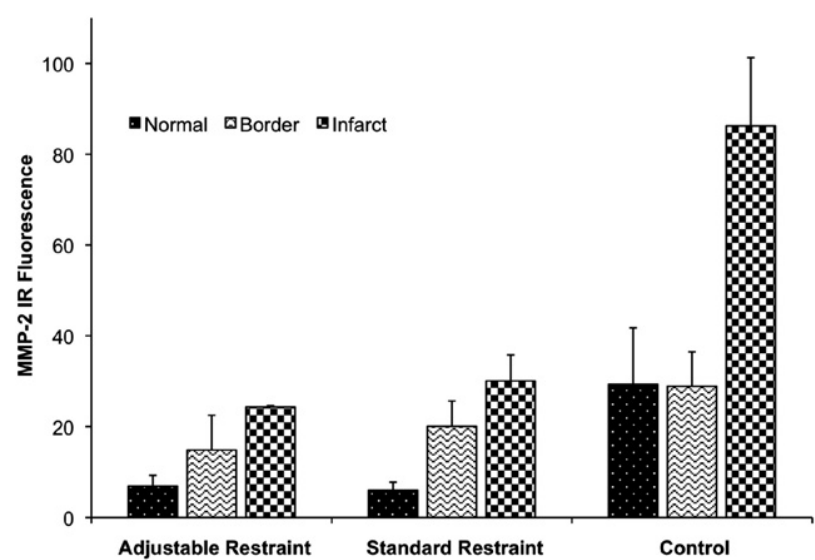

FIGURE 6. Myocardial matrix metalloproteinase-2 (MMP-2) levels in the normal, border, and infarct zones for the adjustable, standard, and control groups. MMP-2 levels are highest in the infarct zone for all subjects. MMP-2 levels are significantly higher in the control group than either restraint treatment group. $I R$, Infrared. such as LV transmyocardial pressure and indices of myocardial oxygen consumption, with higher restraint levels leading to greater decreases in these parameters. Do such changes in ventricular mechanical indices, however, translate into similar changes in the amount of reverse remodeling? No study has yet evaluated the relationship between the amount of applied restraint and the degree of ventricular remodeling. We sought to address that question in this study.

The results from part I demonstrate that with fixed, nonadjusted restraint, the initial restraint level significantly affects both the degree and rate of reverse remodeling. Higher restraint level leads to greater and earlier reductions in $\mathrm{LV}$ EDV compared with the lower restraint level (Figure 2). Why would higher restraint be more effective? So long as tamponade is avoided, greater restraint leads to greater reductions in LV transmyocardial pressure and indices of myocardial oxygen consumption. ${ }^{7,8}$ This implies a greater reduction in ventricular wall stress, which, by extension, would cause greater reverse remodeling. Higher restraint levels apply more pressure on the epicardium not only at end-diastole but also through diastolic filling. ${ }^{7}$ We postulate that this prolonged exposure to higher epicardial pressure promotes increased cellular processes to induce earlier reverse remodeling. Such molecular alterations have been observed in other situations where positive, nontamponade epicardial pressure is applied, such as continuous positive airway pressure therapy for sleep apnea. ${ }^{14}$

What happens to restraint level as the heart shrinks? Not surprisingly, as reverse remodeling occurs and the LV decreases in size, there is a corresponding reduction in the measured restraint level. Inasmuch as fluid is neither added nor removed from the AMVR balloon, the shrinking heart causes the restraint device to effectively loosen. As the effective restraint level decreases, this results in a diminishing epicardial pressure driving the reverse remodeling process forward and reverse remodeling itself slows. In other words, reverse remodeling activity peaks and then declines with concomitant peaks and declines in restraint level. This may help explain clinical data findings in which reverse remodeling slows in patients after 3 months of therapy with fixed, nonadjustable restraint wraps. ${ }^{11,12}$ We conclude that the rate of reverse remodeling for an unchanging restraint device is a function of both the initial restraint level and therapy time. Indeed, the relationship between rate of reverse remodeling and therapy time appears to be nonconstant and nonlinear (Figure 3 ) and doubtless is a dynamic interaction between ventricular size, remodeling, and measured restraint level.

On the basis of these findings, we hypothesized that periodic adjustment of the restraint device to maintain a therapeutic restraint level throughout treatment would be superior to therapy applied in a standard, nonadjusted manner. We postulated that by maintaining a desired restraint pressure (ie, by intermittently adjusting the fluid volume within the AMVR 
device), therapy would be optimized by eliminating the reduction in restraint level caused by the progression of reverse remodeling. This approach would hypothetically apply a continuing impetus to drive forward reverse remodeling even in the face of decreasing ventricular size.

This study demonstrates that adjustable restraint leads to greater reverse remodeling than standard, nonadjustable restraint. LV EDV decreased by approximately $6 \%$ with standard restraint, consistent with results seen in clinical studies of mesh-like restraint wraps. ${ }^{11}$ Adjustable restraint, however, led to a nearly twofold greater improvement with a reduction in LV EDV of approximately $13 \%$. To assess for any effect of animal growth during the treatment period, ventricular volume data was indexed to body weight (which ranged from $40 \mathrm{~kg}$ at the start of the study to $65 \mathrm{~kg}$ at the end of the study). The adjustable group showed greater improvement in these indexed values than did the standard restraint group $(P<.05)$. This suggests that as the heart undergoes reverse remodeling and shrinks, serial measurement and adjustment of the restraint device to maintain the optimal restraint level leads to enhanced therapeutic benefit.

Does restraint therapy cause decreased LV EDV and LV ESV at the expense of hemodynamic output? In this study, cardiac output was not compromised with either adjustable or standard restraint. Rather, cardiac output and stroke volume were increased in both groups at the end of the study period, implying that the observed reduction in ventricular volumes was not associated with hemodynamic decompensation but rather indicative of a true beneficial reverse remodeling process. Possible adverse effects on coronary blood flow can be a concern with excessive epicardial constriction. Large animal studies have shown that application of a meshlike restraint device at levels of up to $2.5 \mathrm{~mm} \mathrm{Hg}$ can be safely applied without significant detrimental effects on coronary blood flow. ${ }^{15}$ This corresponds with our current and previous findings of beneficial changes with restraint up to $3 \mathrm{~mm} \mathrm{Hg}$ without adverse hemodynamic effects.

The degree of maximal improvement in LV EDV in part I ( $28 \%$ in the high restraint group) was different from that found in part II (13\% in the adjustable group). We attribute this difference to the different imaging modalities used: transthoracic echocardiography and CMR. These imaging techniques vary greatly in the methodology of measuring and quantifying ventricular dimensions, which could explain the difference in observed maximal change in ventricular volumes.

We observed changes in molecular markers of heart failure that further confirm the observed morphologic and functional changes. Serum BNP rose, as expected, with the development of heart failure after coronary artery ligation. BNP levels then fell with the initiation of restraint therapy. There was no significant difference between the adjustable and standard restraint groups. BNP measurements alone may not be sufficient to use as a quantitative guide to titrate therapy but could play an adjunctive role in combination with other monitoring parameters.

Myocardial tissue MMP plays a role in cardiac remodeling after myocardial infarction, particularly in the region of ischemia, and serum levels of MMP-2 have been correlated with degree of heart failure. ${ }^{16-19}$ In our study, we found the infarct zone had the highest levels of MMP-2 in all subjects regardless of treatment status. MMP-2 levels were significantly lower in the 2 treatment groups (adjustable restraint and standard restraint) than in control subjects. Between the 2 restraint therapy groups, the adjustable group displayed lower MMP-2 levels than the standard group, although this difference was not statistically significant. Nonetheless, our findings do suggest that restraint therapy in general leads to less MMP-associated remodeling and provides one possible mechanism by which restraint engenders reverse remodeling at the molecular level.

This study does have several limitations. In part I, there were relatively small numbers of animals in each group. Yet, despite the low subject numbers, the study was sufficiently powered to demonstrate statistically significant results. The animal model used was a sheep model of LV postinfarction heart failure and remodeling, and our data should be interpreted with this in mind. Effects of ventricular restraint therapy may be different in heart failure caused by other etiologies or in right heart failure. Another potential weakness that merits future investigation is the duration of therapy. The current project was composed of 4-month studies with treatment times of 2 months. Additional studies must address issues such as the maximum extent of reverse remodeling that is possible and whether pathologic remodeling recurs after termination of restraint therapy.

We used ventricular geometry and size as a marker for ventricular reverse remodeling. This is an accepted technique used clinically for patients with heart failure. An important and useful adjunct, however, would have been direct measurements of cardiac chamber pressures with corresponding pressure-volume analyses. Our group ${ }^{7,8}$ has previously published acute pressure-volume analyses in an identical ischemic heart failure model with the AMVR device. Those studies served as the foundation for the current study, and those data would strongly suggest that had they been measured, the corresponding hemodynamic measurements for this study would be similarly improved.

Conceptually, ventricular restraint therapy is simply controlled and beneficial cardiac constriction at pretamponade levels where systemic hemodynamics are not adversely affected. Restraint level should be titrated to enhance reverse remodeling without creating tamponade. Within the parameters of this physiologic framework, this study shows that restraint level is intimately correlated with the subsequent reverse remodeling. To maximize therapeutic efficacy, 
objective criteria should be established regarding the precise method of restraint application and adjustment for any individual patient. Clinically tested restraint devices do not adequately allow for this. Adjustable and measureable restraint eliminates these shortcomings by potentiating therapy that is more effective than and superior to standard restraint.

Our results demonstrate that by adjusting and measuring restraint level both at the beginning of therapy and as the heart shrinks during active reverse remodeling, restraint therapy can be optimized for improved therapeutic reverse remodeling. We submit that measurable and adjustable ventricular restraint techniques, combined with hemodynamic guides such as real-time echocardiography, could ultimately determine the optimal restraint level for any given individual at any treatment interval. As the heart reverse remodels and restraint level decreases, periodic adjustment of the restraint device would then optimize and enhance subsequent reverse remodeling for improved therapeutic efficacy.

We thank Dr William G. Stetler-Stevenson of the Center for Cancer Research at the National Cancer Institute/National Institutes of Health for providing the rabbit anti-MMP-2 antibody, and Ethicon US (Somerville, NJ) for providing the polypropylene mesh used in this study.

\section{References}

1. Oh JH, Badhwar V, Mott BD, Li CM, Chiu RC. The effects of prosthetic cardiac binding and adynamic cardiomyoplasty in a model of dilated cardiomyopathy. $J$ Thorac Cardiovasc Surg. 1998;116:148-53.

2. Konertz WF, Shapland JE, Hotz H, Dushe S, Braun JP, Stantke K, et al. Passive containment and reverse remodeling by a novel textile cardiac support device. Circulation. 2001(12 Suppl 1);104:I270-5.

3. Power JM, Raman J, Dornom A, Farish SJ, Burrell LM, Tonkin AM, et al. Passive ventricular constraint amends the course of heart failure: a study in an ovine model of dilated cardiomyopathy. Cardiovasc Res. 1999;44:549-55.

4. Sabbah HN, Sharov VG, Gupta RC, Mishra S, Rastogi S, Undrovinas AI, et al. Reversal of chronic molecular and cellular abnormalities due to heart failure by passive mechanical ventricular containment. Circ Res. 2003;93:1095-101.

5. Pilla JJ, Blom AS, Brockman DJ, Ferrari VA, Yuan Q, Acker MA. Passive ventricular constraint to improve left ventricular function and mechanics in an ovine model of heart failure secondary to acute myocardial infarction. J Thorac Cardiovasc Surg. 2003;126:1467-76.

6. Cheng A, Nguyen TC, Malinowski M, Langer F, Liang D, Daughters GT, et al. Passive ventricular constraint prevents transmural shear strain progression in left ventricle remodeling. Circulation. 2006;114(1 Suppl):I79-86.

7. Ghanta RK, Rangaraj A, Umakanthan R, Lee L, Laurence RG, Fox JA, et al. Adjustable, physiological ventricular restraint improves left ventricular mechanics and reduces dilatation in an ovine model of chronic heart failure. Circulation. 2007;115:1201-10

8. Lee LS, Ghanta RK, Mokashi SA, Coelho-Filho O, Kwong RY, Bolman RM III, et al. Ventricular restraint therapy for heart failure: the right ventricle is different from the left ventricle. J Thorac Cardiovasc Surg. 2010;139:1012-8.

9. Moainie SL, Gorman JH 3rd, Guy TS, Bowen FW 3rd, Jackson BM, Plappert T, et al. An ovine model of postinfarction dilated cardiomyopathy. Ann Thorac Surg. 2002;74:753-60.

10. Oz MC, Konertz WJ, Raman J, Kleber FX. Reverse remodeling of the failing ventricle: surgical intervention with the Acorn cardiac support device. Congestive Heart Fail. 2004;10:96-104.

11. Oz MC, Konertz WF, Kelber FX, Mohr FW, Gummert JF, Ostermeyer J, et al. Global surgical experience with the Acorn cardiac support device. J Thorac Carciovasc Surg. 2003;126:983-91.

12. Klodell CT Jr, McGiffin DC, Rayburn BK, Sun B, Abraham WT, Conte JV, et al Initial United States experience with the Paracor HeartNet myocardial constraint device for heart failure. J Thorac Cardiovasc Surg. 2007;133:204-9.

13. Starling RC, Jessup M, Oh JK, Sabbah HN, Acker MA, Mann DL, et al. Sustained benefits of the CorCap Cardiac Support Device on left ventricular remodeling: three year follow-up results from the Acorn clinical trial. Ann Thorac Surg. 2007;84:1236-42.

14. Chin K, Nakamura T, Shimizu K, Mishima M, Nakamura T, Miyasaka M, et al Effects of nasal continuous positive airway pressure on soluble cell adhesion molecules in patients with obstructive sleep apnea syndrome. Am J Med. 2000; 109:562-7.

15. Dixon JA, Goodman AM, Gaillard WF, Rivers WT, McKinney RA, Mukherjee R, et al. Hemodynamics and myocardial blood flow patterns after placement of a cardiac passive restraint device in a model of dilated cardiomyopathy. J Thorac Cardiovasc Surg. 2011;142:1038-45.

16. Rohde LE, Ducharme A, Arroyo LH, Aikawa M, Sukhova GH, Lopez-Anaya A, et al. Matrix metalloproteinase inhibition attenuates early left ventricular enlargement after experimental myocardial infarction in mice. Circulation. 1999; 99:3063-70.

17. Yamazaki T, Lee JD, Shimizu H, Uzui H, Ueda T. Circulating matrix metalloproteinase-2 is elevated in patients with congestive heart failure. Eur $J$ Heart Fail. 2004;6:41-5.

18. Shirakabe A, Asai K, Hata N, Yokoyama S, Shinada T, Kabayashi N, et al. Prognostic impact of matrix metalloproteinases in patients with acute heart failure. $J$ Am Coll Cardiol. 2010;55:A34.E330.

19. Creemers E, Cleutjens J, Smits J, Daemen M. Matrix metalloproteinase inhibition after myocardial infarction. A new approach to prevent heart failure? Circ Res. 2001;89:201-10 